\title{
CARACTERÍSTICAS SOCIODEMOGRÁFICAS Y ESTILOS DE VIDA EN ESTUDIANTES DE ENFERMERÍA UNIVERSIDAD NACIONAL SAN LUIS GONZAGA DE ICA 2014.
}

\author{
Socio-Demographic Characteristics and lifestyles in students of National University San Luis \\ Gonzaga de Ica 2014 \\ Marianela Clarisa Pecho Tataje ${ }^{1 a, b}$, Cecilia Paquita Uribe Quiroz ${ }^{1 a, b}$,Viviana Loza Félix ${ }^{2 a}$ \\ 1 Hospital Augusto Hernández Mendoza \\ 2 Facultad de enfermería de la Universidad Nacional San Luis Gonzaga de Ica, Perú \\ a Lic. en enfermería b Mg. En Educación Mención en Administración y Planificación de la Educación Superior.
}

\section{Resumen}

Los estilos de vida a lo largo de la historia son considerados determinantes de mayor predominio en el proceso salud enfermedad, esto significa que su abordaje es de vital importancia en todas las etapas de vida. El objetivo del estudio fue determinar la relación entre las características sociodemográficas y los estilos de vida de los estudiantes de enfermería de la Universidad Nacional San Luis Gonzaga de Ica 2014. Material y Métodos: Se tomó en cuenta una muestra de 176 estudiantes cantidad obtenida por muestreo probabilístico estratificado, teniendo como técnica la encuesta cuyo instrumento fue un cuestionario validado y confiable. El tipo de estudio fue descriptivo correlacional, de enfoque cuantitativo y de corte transversal. Resultados: Dentro de las características sociodemográficas de los estudiantes se encontró que viven con ambos padres $(58,12 \%)$, su relación familiar es buena $(66,49 \%)$; no tienen hijos $(87,43 \%)$; solo estudian $(82,72 \%)$; dependen económicamente de sus padres (76,44\%); la persona de quien dependen económicamente tiene un trabajo estable $(69,11 \%)$ y tienen un ingreso económico mensual de S/. 1500 a S/. 2000 (45,03\%); la edad fue 16 y 19 años (50\%), sexo femenino (93\%); solteros $(97 \%)$ y su procedencia fue Ica (72\%). Los estilos de vida fueron no saludables en el $57 \%$ de los estudiantes. Conclusiones: Se demostró que existe relación entre las características sociodemográficas y los estilos de vida de los estudiantes de enfermería de la Universidad Nacional San Luis Gonzaga de Ica. $\left(X^{2}=3,56\right)$.

Palabras clave: Características sociodemográficas, estilo de vida, estudiante universitario

\section{SUMMARY}

Lifestyles throughout history are considered to be determinants of greater prevalence in the disease health process, this means that their approach is of vital importance in all stages of life. The objective of the study was to determine the relationship between the sociodemographic characteristics and the lifestyles of the nursing students of the National University San Luis Gonzaga de Ica 2014. Material and Methods: A sample of 176 students was taken into account amount obtained by sampling probabilistic stratified, having as a technique the survey whose instrument was a validated and reliable questionnaire. The type of study was descriptive correlational, with a quantitative and cross-sectional approach. Results: Within the sociodemographic characteristics of the students, it was found that they live with both parents $(58.12 \%)$, their family relationship is good $(66.49 \%)$; they do not have children $(87.43 \%)$; they only study (82.72\%); they depend economically on their parents $(76.44 \%)$; the person on whom they depend economically has a stable job (69.11\%) and they have a monthly income of $S / .1500$ to S /. 2000 (45.03\%); the age was 16 and 19 years old (50\%), female sex (93\%); singles (97\%) and their origin was Ica (72\%). Lifestyles were unhealthy in $57 \%$ of students. Conclusions: It was demonstrated that there is a relationship between the sociodemographic characteristics and lifestyles of the nursing students of the National University of San Luis Gonzaga de Ica. (X2 = 3.56). 
Key words: Sociodemographic characteristics, lifestyle, university student

\section{INTRODUCCIÓN}

La vida universitaria implica organizar el tiempo del estudiante en labores académicas, las mismas que pueden ser teóricas o prácticas sobre todo en las carreras de salud; las conductas adquiridas en etapas anteriores sufren cambios que involucran su vida personal y familiar, de los cuales se forman estilos de vida que determinan la conservación de la salud actual y en el futuro.

La Organización Mundial de la Salud ha establecido diversos planes de acción que ayuden a reducir la tasa de morbimortalidad de enfermedades no trasmisibles originadas principalmente por los estilos de vida no saludables, dentro de las que se menciona la obesidad, diabetes mellitus, hipertensión, enfermedades cardiovasculares e incluso aquellas enfermedades oncológicas(1).

$\begin{array}{ccr}\text { Un } & \text { reciente } & \text { estudio } \\ \text { epidemiológico } & \text { analítico } & \text { acumula }\end{array}$ evidencias de los efectos positivos y negativos de la actividad física en la prevención de diversas enfermedades, reduciendo el riesgo de muerte. Además, la promoción de estilos de vida saludables en el ámbito universitario es escaso, pues, pareciera que, el hecho de encontrarse en un nivel superior sus necesidades académicas acaparan toda la atención de la persona y las instituciones (2).

Los estilos de vida incluyen diversas conductas considerados principalmente por Pender como esenciales en la conservación de la salud, tales como: Alimentación, Actividad física, Descanso y sueño, higiene, relaciones interpersonales que, además, posibilitan la autorrealización personal (3). De esta manera, diversas investigaciones confirman que, adoptar un estilo de vida físicamente activo se asocia, entre otras cosas, con un menor riesgo de hipertensión arterial, cardiopatía isquémica, osteoporosis, obesidad, diabetes y otras enfermedades y afecciones tales como la modificación del nivel de tolerancia a la glucosa, de la sensibilidad a la insulina o del perfil lipídico así como, ansiedad, insomnio, trastornos del ánimo e incluso, menor riesgo de sufrir cáncer (4).

En un estudio realizado en Argentina, respecto a los estilos de vida en universitarios destaca que existe una alta prevalencia de prácticas no saludables en actividad física (82\%); autocuidado y control médico (64\%) e incluso la alimentación (52\%) condicionando un bajo nivel de autoconcepto según su estado físico principalmente en las mujeres (41\%) (5).

Dicha problemática no es ajena al Perú, tal es así que un estudio epidemiológico realizado por el Instituto Nacional de Salud mental Honorio Delgado en el 2011 señala que el 7,3 y $11,4 \%$ de más de nueve mil adolescentes y jóvenes encuestados en hogares de 17 ciudades, sufren problemas alimentarios, estas enfermedades comprometen el desarrollo normal de los jóvenes debido a cambios de sus hábitos alimenticios y actividades en general, lo que genera en consecuencia complicaciones físicas y mentales(6).

En Lima una investigación revela que el $55 \%$ de los estudiantes universitarios de 
enfermería; en la dimensión biológica, donde se incluyeron hábitos alimenticios, actividad física, ocio y horario de sueño el $51 \%$ tienen estilos de vida desfavorables; en la dimensión social el $25 \%$ tienen estilos de vida desfavorables(7).

Los estilos de vida de una población son considerados uno de los determinantes del proceso salud enfermedad, de allí la importancia de su abordaje. La identificación del estilo de vida que tiene el estudiante universitario sentará las bases para el trabajo conjunto en el logro de universidades saludables. Asimismo, la percepción de su autorrealización complementada en la universidad puede relacionarse a su salud mental, la cual constituye uno de los ejes de la promoción de salud.

La Universidad Nacional San Luis Gonzaga de Ica (UNICA)alberga estudiantes de la región e incluso de regiones de la serranía de mayor acercamiento, la facultad de enfermería corresponde a una de las carreras profesionales de mayor elección en los últimos años, por la naturaleza de la profesión, los estudiantes de enfermería de la UNICA, cumplen horarios completos en la mañana y por la tarde, situación que constituye un obstáculo a favor de las conductas saludables, dado que, muchos de los estudiantes alteran sus horarios de comida o lo realizan de paso entre un lugar $y$ otro, las exigencias académicas y los propios adelantos tecnológicos ejercen presión en la búsqueda de información frente a un computador reemplazando ciertas actividades físicas por otras sedentarias, asimismo manifiestan menor cantidad de horas de sueño, descuido en el control de la salud entre otros aspectos.

El objetivo fue determinar la relación entre las características sociodemográficas y los estilos de vida de los estudiantes de enfermería de la Universidad Nacional San Luis Gonzaga de Ica 2014.

\section{MATERIAL Y MÉTODOS:}

El estudio es de enfoque cuantitativo, alcance correlacional de corte transversal, tomó en cuenta una muestra de 191 estudiantes, cantidad obtenida por muestreo probabilístico estratificado incluyendo a los estudiantes de I a VIII ciclo por tener características homogéneas. La técnica de recolección de datos fue la encuesta cuyo instrumento fue un cuestionario de opción múltiple para las características sociodemográficas con un total de 10 preguntas y una escala de Likert en tres valores para los estilos de vida con 33 ítems, instrumento validado y trabajado en poblaciones similares por Cuba (2010). La aplicación del instrumento se hizo tomando en cuenta aspectos éticos como el respeto a la dignidad humana, autonomía del participante y confidencialidad.

\section{RESULTADOS}

Al relacionar los estilos de vida con las características sociales se encontró que los estudiantes que tenían mayor porcentaje de estilos de vida no saludables fueron aquellos que vivían con sus padres $29,3 \%$ (56 estudiantes), además los estudiantes que solo estudian tienen mayor proporción de estilos de vida no saludables $43,5 \%$ (83 estudiante. 
Tabla 1: Relación entre los Estilos de vida y las Características Sociales de los Estudiantes de la Facultad de Enfermería Universidad Nacional San Luis Gonzaga de Ica 2014

\begin{tabular}{|c|c|c|c|c|c|c|c|}
\hline \multirow{3}{*}{$\begin{array}{l}\text { CARACTERISTICAS } \\
\text { SOCIALES }\end{array}$} & \multicolumn{7}{|c|}{ ESTILOS DE VIDA } \\
\hline & & \multicolumn{2}{|c|}{ SALUDABLES } & \multicolumn{2}{|c|}{ SALUDABLES } & \multicolumn{2}{|c|}{ TOTAL } \\
\hline & CATEGORIAS & $\mathrm{f}$ & $\%$ & $\mathrm{f}$ & $\%$ & $\mathrm{f}$ & $\%$ \\
\hline & $\begin{array}{l}\text { AMBOS } \\
\text { PADRES }\end{array}$ & 55 & 28,80 & 56 & 29,32 & 111 & 58,12 \\
\hline & $\begin{array}{l}\text { SOLO UNO DE } \\
\text { ELLOS }\end{array}$ & 20 & 10,47 & 20 & 10,47 & 40 & 20,94 \\
\hline & HERMANOS & 1 & 0,52 & 5 & 2,62 & 6 & 3.14 \\
\hline & ABUELOS & 3 & 1,57 & 6 & 3,14 & 9 & 4,71 \\
\hline & PERSONA NO & 5 & 2,62 & 20 & 10,47 & 25 & 13,09 \\
\hline VIVE CON & FAMILIAR & & & & & & \\
\hline & ESTUDIA Y & $\begin{array}{r}10 \\
9\end{array}$ & $\begin{array}{r}39,21 \\
471\end{array}$ & 24 & $\begin{array}{l}43,46 \\
1257\end{array}$ & 33 & $\begin{array}{l}82, / 2 \\
17,28\end{array}$ \\
\hline OCUPACION & TRABAJA & & & & & & \\
\hline & $A L$ & 84 & 43,98 & 107 & 5602 & 191 & 100,0 \\
\hline
\end{tabular}

Tabla 2: Relación entre los Estilos de Vida y las Características Sociales de los Estudiantes de la Facultad de Enfermería Universidad Nacional San Luis Gonzaga de Ica 2014

\begin{tabular}{|c|c|c|c|c|c|c|c|}
\hline \multirow{3}{*}{$\begin{array}{c}\text { CARACTERISTICAS } \\
\text { DEMOGRÁFICAS }\end{array}$} & \multicolumn{7}{|c|}{ ESTILOS DE VIDA } \\
\hline & \multirow[b]{2}{*}{ CATEGORIAS } & \multicolumn{2}{|c|}{ SALUDABLES } & \multicolumn{2}{|c|}{ NO SALUDABLES } & \multicolumn{2}{|c|}{ TOTAL } \\
\hline & & $\mathbf{f}$ & $\%$ & $\mathbf{f}$ & $\%$ & $\mathbf{f}$ & $\%$ \\
\hline & 16 a 19 años & 30 & 15,71 & 65 & 34,03 & 95 & 49,74 \\
\hline & 20 a 23 años & 46 & 24,08 & 40 & 20,94 & 86 & 45,03 \\
\hline \multirow[t]{2}{*}{ EDAD } & 24 a 27 años & 8 & 4,71 & 2 & 1,05 & 10 & 5,24 \\
\hline & Masculino & 10 & 5,24 & 4 & 2,09 & 14 & $7.33 \%$ \\
\hline \multirow[t]{6}{*}{ SEXO } & Femenino & 74 & $38.74 \%$ & 103 & $53.93 \%$ & 177 & $92.67 \%$ \\
\hline & Ica & 55 & $28.80 \%$ & 83 & $43.46 \%$ & 138 & $72.25 \%$ \\
\hline & Provincias de Ica & 12 & $6.28 \%$ & 8 & $4.19 \%$ & 20 & $10.47 \%$ \\
\hline & Sierra & 12 & $6.28 \%$ & 11 & $5.76 \%$ & 23 & $12.04 \%$ \\
\hline & Lima & 3 & $1.57 \%$ & 4 & $2.09 \%$ & 7 & $3.66 \%$ \\
\hline & Selva & 2 & $1.05 \%$ & 0 & $0.0 \%$ & 2 & $1.05 \%$ \\
\hline PROCEDENCIA & Costa norte & 0 & $0.0 \%$ & 1 & $0.52 \%$ & 1 & $0.52 \%$ \\
\hline \multicolumn{2}{|c|}{ TOTAL } & 84 & $44.0 \%$ & 107 & $56.02 \%$ & 191 & $100.0 \%$ \\
\hline
\end{tabular}

Al relacionar los estilos de vida con las características demográficas como la edad se obtuvo que los estudiantes con mayor proporción de estilos de vida no saludables están en los adolescentes quienes tenían entre 16 a 19 años $34.03 \%(65)$; respecto al sexo, la mayor proporción de estilos de vida no saludables corresponden al femenino $53.93 \%(103)$, asimismo, los estudiantes que eran de la provincia de Ica tenían estilos de vida no saludables en $43.46 \%(83)$ 
Tabla No 3

ESTILOS DE VIDA SEGÚN DIMENSIONES DE LOS ESTUDIANTES DE ENFERMERÍA DE LA UNICA 2014.

\begin{tabular}{lrrrrrr}
\hline $\begin{array}{l}\text { Estilos de vida según } \\
\text { dimensiones }\end{array}$ & \multicolumn{2}{c}{$\begin{array}{c}\text { Saludables } \\
\mathrm{f}\end{array}$} & $\%$ & $\mathrm{f}$ & & \multicolumn{2}{c}{ Total } \\
\hline $\begin{array}{l}\text { Alimentación } \\
\text { Actividades lúdicas y }\end{array}$ & 86 & $45 \%$ & 105 & $55 \%$ & 191 & $100 \%$ \\
físicas & 89 & $47 \%$ & 102 & $53 \%$ & 191 & $100 \%$ \\
$\begin{array}{l}\text { Relaciones } \\
\text { interpersonales }\end{array}$ & 122 & $64 \%$ & 69 & $36 \%$ & 191 & $100 \%$ \\
Higiene & 136 & $71 \%$ & 55 & $29 \%$ & 191 & $100 \%$ \\
$\begin{array}{l}\text { Sueño y descanso } \\
\text { Cuidado de la salud }\end{array}$ & 0 & $0 \%$ & 191 & $100 \%$ & 191 & $100 \%$ \\
\hline
\end{tabular}

\section{DISCUSIÓN}

Las características sociales de los estudiantes de enfermería (Tabla 1) fueron vivir con ambos padres $58,12 \%$; un $66,49 \%$ consideraron que la relación familiar fue buena, un $12,57 \%$ tienen hijos; tienen como ocupación solo estudiar 82,72\%; dependen económicamente de sus padres $76,44 \%$; la persona de quien dependen económicamente tiene un trabajo estable $69,11 \%$ y el $45,03 \%$ tienen un ingreso económico mensual de S/. 1500 a 2000.

Resultados que tienen similitud con los de Páez M, Castañeda J.(8),en cuyo estudio manifiesta que la relación en su mayoría muy estrecha solo con la familia, sin embargo, resalta un 62,9\% de disfunción familiar, entre leve y severa. Si se evalúan dichas características se resalta la existencia de situaciones favorables en la mayoría de ellas, que un estudiante universitario tenga buena relación familiar y solo se dedique a estudiar, son condiciones que favorecen el cuidado de la salud, sin embargo la vida universitaria y sobre todo en el campo de la salud como es enfermería implica la existencia de aprendizaje teórico práctico, lo cual en gran proporción del ciclo académico amerita doble horario: mañana (práctica clínica) y tarde (clases teóricas) involucra absorción de tiempo completo, creando un desequilibrio en el ambiente del adolescente o joven estudiante.

Es positivo mencionar que la institución donde se realiza el estudio es pública, lo cual implica costo mínimo, sobre todo considerando que los ingresos económicos familiares de los estudiantes solo superan la barrera del sueldo mínimo vital que como se sabe no permite cubrir la canasta familiar.

Al relacionar los estilos de vida con las principales características sociales, se encontró que los estudiantes que tenían mayor porcentaje de estilos de vida no saludables fueron aquellos que vivían con sus padres $29,3 \%$, además los estudiantes que solo estudian tienen mayor proporción de estilos de vida no saludables $43,5 \%$. Resultados que guardan similitud con el estudio de Bennasar M.(9), quien observa que son determinantes de una dieta saludable, el realizar ejercicio físico, no fumar, desayunar todos los días y realizar un mayor número de comidas. Los 
resultados reafirman la idea de que los comportamientos de salud no deben de considerarse de forma aislada unos de otros. Todo el entorno es significativo para la persona, considerando que el ser humano es un ente social, siempre está en proceso de aprendizaje de una nueva experiencia sobre todo cuando se reúnen con sus pares.

Dentro de los datos demográficos de los estudiantes de enfermería (Tabla 2) se encontró que tenían edades entre 16 y 19 años en 50\%; el sexo femenino alcanzó 93\%; su estado civil fue soltero en el $97 \%$ y su procedencia fue Ica en un $72 \%$. Resultados que difieren a los de Páez M, Castañeda J.(10), en cuya investigación encontró que la edad promedio de su población fue 21,42 años; sin embargo guardan similitud con el género encontrando un $82 \%$ femenino y el estado civil soltero quienes alcanzaron $85,4 \%$ de los resultados se destaca la existencia de estudiantes en etapa de vida adolescente, etapa caracterizada por cambios tanto físicos como emocionales que pueden interferir en la adquisición de conductas, las mismas que van a determinar el cuidado de la salud presente y futura. Además, como sucede hasta el momento la mayoría de mujeres abarcan estudios de enfermería, siendo una profesión eminentemente de servicio, que hasta el momento tenga la condición de soltero y sin hijos se convierte en una fortaleza porque su dedicación esta direccionada mayormente hacia su formación profesional.

El aspecto de las conductas o comportamientos es decisivo en el estado de bienestar de las personas, tanto en el plano físico como psicológico. $Y$ es necesario considerar que la conducta obedece a múltiples factores que interactúan de manera dinámica y diferencialmente según la situación: los valores, creencias, expectativas, conocimientos, actitudes, la familia y sociedad; y otros elementos más forman parte de estos factores condicionantes en los estudiantes universitarios.

Al relacionar los estilos de vida con las características demográficas (Tabla 3) como la edad se obtuvo que los estudiantes con mayor proporción de estilos de vida no saludables están en los adolescentes quienes tenían entre 16 a 19 años 34,03\%; respecto al sexo, la mayor proporción de estilos de vida no saludables corresponden al femenino 53,93\%, asimismo, los estudiantes que eran de la provincia de Ica tenían estilos de vida no saludables en $43,46 \%$. Además, se puede considerar, a pesar de no constituir una cantidad abrumadora, que los varones de manera global tienen mejores estilos de vida que las mujeres. Al respecto Piscicelli A. (10), en su investigación menciona que la dimensión física, la mayoría de los varones presento un nivel normal 0 esperable de autoconcepto (59\% de los varones) y en las mujeres, por el contrario, la mayoría un nivel bajo de autoconcepto $(41 \%$ de las mujeres). Esto evidencia disconformidad con el logro de metas, búsqueda de sentido, entre otros aspectos.

Los estilos de vida de los estudiantes de enfermería según sus dimensiones: alimentación, actividades lúdicas y físicas; sueño y descanso y cuidado de la salud (Tabla 3) fueron no saludables alcanzando 55\%; 53\%; $100 \%$ y $57 \%$ respectivamente, sin embargo, respecto a relaciones interpersonales e higiene fueron saludables en $64 \%$ y $71 \%$ para cada dimensión. Resultados que tienen similitud en algunas dimensiones como 
alimentación, actividad física y relaciones interpersonales, con Ponte M.(11), quien en su investigación obtuvo resultados que al analizarlo por dimensiones; se tiene que, en la dimensión actividad física con 82,9\% predomina un estilo de vida no saludable; en la dimensión nutrición saludable el $65,2 \%$ tienen un estilo de vida no saludable; y relaciones interpersonales $75,2 \%$ predomina el estilo de vida saludable en los cinco años de estudio.

A pesar de las evidentes ventajas, la práctica de ejercicio físico no es tan frecuente entre los jóvenes y, especialmente los universitarios- como sería deseable, considerando que cuentan con tiempo completo de carga académica, lo cual incluye horarios en turno mañana para labores de práctica clínica o de campo y el turno tarde para el desarrollo de labores teóricas en las diferentes materias de especialidad y otras áreas, este misma carga académica imposibilita consolidar horarios adecuados para el consumo de alimentos, horas de sueño y descanso, necesarias para la conservación del estado de salud.

Las relaciones interpersonales y hábitos de higiene también forman parte de los estilos de vida, lo cual posibilita el desarrollo personal, considerando que la mayoría es adolescente además de la conservación de la salud. Una de las herramientas fundamentales de la promoción de la salud es la educación para la salud, que aborda no solamente la transmisión de información, sino también el fomento de la motivación, las habilidades personales y la autoestima, necesarias para adoptar medidas destinadas a mejorar la salud.

El cuidado de la salud de los estudiantes de enfermería considerada como una dimensión aborda la responsabilidad que tienen los estudiantes conductas que lleven a cabo para conservar su salud dentro de las cuales está el realizar control médico, clínico, existencia de enfermedades que ameriten tratamiento y control continuo, existencia de hábitos nocivos, e incluso conductas sexuales, en tal sentido los resultados de la dimensión evidencian ser no saludables. Resultados que difieren a los de Ponte M. (11), en cuya investigación obtuvo resultados que muestran que el $60 \%$ de los estudiantes tiene un estilo de vida saludable; al analizarlo por dimensiones; se tiene que, en la dimensión salud con responsabilidad con $76,7 \%$. Sin embargo, Páez M, Castañeda J. (8), en su investigación identificó un $65,1 \%$ de no fumadores; $26,9 \%$ no consumen alcohol; concluyendo que los altos indicadores de factores de riesgo para la salud en esta población confirman lo descrito en otras investigaciones efectuadas con jóvenes universitarios.

Al realizar la prueba de correlación entre las variables, se obtuvo de acuerdo al estadístico chi cuadrado que No existe relación entre las características sociodemográficas y los estilos de vida de los estudiantes de enfermería de la UNICA 2014. Resultados similares a los de Gómez J. (12), quien en su investigación determinó que no existe correlación entre calidad de vida y actividad deportiva.

La promoción de la salud en la Universidad será un valor añadido para la mejora de la calidad de vida y el bienestar de los que allí estudien o trabajen y además, permitirá formarlos con la finalidad de que actúen como modelos o promotores de conductas saludables en sus familias, en sus futuros entornos laborales, en su comunidad y en la sociedad en general. 
El fomento de la salud consistente hacia la calidad de vida y consecuentemente de la salud, con lleva a mejorar el bienestar del individuo y comunidades, a reducir riesgos y tratar enfermedades. La ausencia de prevención y promoción de la salud, ha desencadenado un aumento en la incidencia de enfermedades cardiovasculares, hipertensión, diabetes, dislipidemia provocando que tanto el recurso físico, como humano sea insuficiente para brindar la atención oportuna y de calidad a la población con la consecuente insatisfacción de los usuarios. La universidad constituye un escenario propicio para dicha estrategia en búsqueda de la conservación de la salud.

\section{CONCLUSIONES}

-No existe relación entre las características sociodemográficas y los estilos de vida de los estudiantes de enfermería de la Universidad Nacional San Luis Gonzaga de Ica 2014, demostrándose que a mejores estilos de vida menor riesgo de enfermar. $\left(\mathrm{chi}^{2}=\right.$ 0.70; p: 0.05)

- La relación entre las características sociodemográficas y estilos de vida de los estudiantes de enfermería refleja que la mayoría de estudiantes que viven con sus padres y solo estudian tienen estilos de vida no saludables; asimismo, los estudiantes más jóvenes (16 a 19 años), de sexo femenino y cuya procedencia era la provincia de Ica, tiene estilos de vida no saludables.

- Los estilos de vida respecto a la alimentación, actividad física; sueño y descanso y cuidado de la salud fueron no saludables en los estudiantes de enfermería de la Universidad Nacional San Luis Gonzaga de Ica, sin embargo, respecto a las relaciones interpersonales e higiene fueron saludables, obteniendo de manera global que los estilos de vida en los estudiantes de enfermería fueron no saludables.

\section{Correspondencia}

Mg. Marianela Clarisa Pecho Tataje

Correo

electrónico:mclarisapecho@gmail.com

\section{REFERENCIAS BIBLIOGRÁFICAS}

1. OMS. Día mundial de la salud. ( Internet) Washington; 2012.

Citado el 12 de Enero 2015)

Disponible en:

http://www.who.int/world-healthday/2012/toolkit/campaign/es/ .

2. Estilos de vida y salud en estudiantes de una facultad de psicología. ( Citado el 2 de Enero 2015) Disponible en: http://www.scielo.org.co/scielo.php ?script=sci_arttext\&pid=s0123417x2010000100008.

3. Raile M. Teorías y modelos de enfermería. $8^{a}$ ed. Elsevier: España; 2015.

4. Sánchez J. evaluación del programa deportivo universitario del servicio de deportes en el campus de Segovia. (Internet) Universidad de Valladolid. España; 2015.(Citado el 20 de Julio 2014) Disponible en: http://uvadoc.uva.es/bitstream/103 24/25453/1/TFM-B.1089.pdf.

5. Sieghart N. Autoconcepto y estilos de vida. Tesis de Pregrado. Argentina. Universidad de FASTA; 2012. 8 Citado el 21 de Noviembre 2014) Disponible en: http://redi.ufasta.edu.ar:8080/xmlui /bitstream/handle/123456789/258/ 2012_n_028.pdf?sequence $=1$. 
6. Ministerio de Salud. Según el instituto nacional de salud mental Honorio delgado (Internet). Perú: MINSA; [actualizada 21 de junio de 2010; (Citado el 2 de Diciembre 2015). Disponible en: http://www.minsa.gob.pe/portada/ prensa/nota completa.asp?nota $=9$ $\underline{146}$

7. Mendoza S. Estilos de vida en estudiantes de enfermería de la Universidad Nacional Mayor de San Marcos. Tesis Pregrado .Lima, Perú. Facultad de Medicina, EAP Enfermería, Universidad Nacional Mayor de San Marcos; 2010.(Citado el 22 de Diciembre 2014) Disponible en: http://biblioteca.universia.net/html_ bura/ficha/params/title/estilosvida-estudiantes-enfermeriauniversidad-nacional-san-marcos2006/id/51067116.html.

8. Páez M, Castaño J. Estilos de vida y salud en estudiantes de una facultad de psicología Universidad del Norte. (Internet). Colombia. Rev. Psicología desde el Caribe.2010; 25(1):155-178. (Citado el 22 de Enero 2015) Disponible en: http://redalyc.uaemex.mx/pdf/213/ 21315106008.pdf.

9. Bennasar M. Estilos de vida y salud en estudiantes universitarios: la universidad como entorno promotor de la salud (Citado el 22 de Enero 2015) Disponible en: http://evesresearch.uib.cat/estilosde-vida-y-salud-en-estudiantesuniversitarios-la-universidadcomo-entorno-promotor-de-lasalud/.

10. Piscicelli, A. Estilo de vida, el nivel de auto concepto y el estado nutricional en alumnos que concurren a una Escuela Pública de Nivel Medio para adultos de la ciudad de Mar del Plata. Tesis de grado Argentina; 2012.

11. Ponte M. Estilo de vida de los estudiantes de Enfermería, según año de estudio de la Universidad Nacional Mayor de San Marcos. Tesis. Lima, Perú. Facultad de Medicina, EAP Enfermería, Universidad Nacional Mayor se San Marcos; 2010.

12. Gómez J, Salazar N. Hábitos alimenticios en estudiantes Universitarios de Ciencias de la Salud. Tesis Pregrado. Minatitlán: México Universidad Veracruzana, 2010. (Citado el 22 de Enero 2015) Disponible en: http://cdigital.uv.mx/bitstream/123 456789/30073/1/GomezDomingue zJlySalazarHdzNayeli.pdf.

Recibido: 02/01/2017 Aprobado para publicación: 15/07/2017 